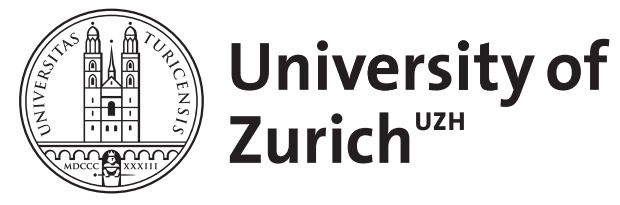

Zurich Open Repository and Archive

University of Zurich

University Library

Strickhofstrasse 39

CH-8057 Zurich

www.zora.uzh.ch

Year: 2009

\title{
Operational risk quantification using extreme value theory and copulas: from theory to practice
}

\author{
Gourier, Elise ; Abbate, Donato ; Farkas, Walter
}

Posted at the Zurich Open Repository and Archive, University of Zurich ZORA URL: https://doi.org/10.5167/uzh-99902

Journal Article

Originally published at:

Gourier, Elise; Abbate, Donato; Farkas, Walter (2009). Operational risk quantification using extreme value theory and copulas: from theory to practice. The Journal of Operational Risk, 4(3):1-24. 


\title{
Operational risk quantification using extreme value theory and copulas: from theory to practice
}

\section{Elise Gourier}

Deloitte AG, Risk and Performance Management Group, General Guisan-Quai 38, 8022 Zurich, Switzerland

and

Swiss Banking Institute, University of Zurich, Plattenstrasse 14, 8032 Zurich, Switzerland; email: gourier@isb.uzh.ch

\section{Walter Farkas}

Swiss Banking Institute, University of Zurich, Plattenstrasse 14, 8032 Zurich, Switzerland; email: farkas@isb.uzh.ch

partly supported by

Deloitte AG Zurich, Risk and Performance Management Group, General Guisan-Quai 38, 8022 Zurich, Switzerland

\section{Donato Abbate}

Deloitte AG, Risk and Performance Management Group, General Guisan-Quai 38, 8022 Zurich, Switzerland; email: dabbate@deloitte.ch

\begin{abstract}
In this paper we perform an empirical study pointing out several pitfalls of the standard methodologies for quantifying operational losses. Firstly, we use extreme value theory to model real heavy-tailed data. We show that using value-at-risk as a risk measure may lead to a misestimation of the capital requirements. In particular, we examine the issues of stability and coherence and relate them to the degree of heavy-tailedness of the data. Secondly, we introduce dependence between the business lines using copula theory. We show that standard economic thinking about risk diversification may be inappropriate when infinite-mean distributions are involved, as is standard in operational risk.
\end{abstract}

\section{INTRODUCTION}

On January 24, 2008, Société Générale, one of the largest banks in Europe, was thrown into turmoil after it revealed that a rogue employee, Jérôme Kerviel, had executed a series of "elaborate, fictitious transactions" that cost the company more than $€ 4.9$ billion, the biggest loss ever recorded in the financial industry by a single trader.

Events similar to that one continue to shake the world's financial markets, and raise the awareness of banks, trading houses and regulatory agencies regarding the inherent operational risks involved in trading operations. 
In an attempt to provide a regulatory framework to handle operational risk, the Basel Committee on Banking Supervision published in 2004 and updated in 2007 a new Basel Accord, which opened the door to operational risk, defined by the Basel Committee of Banking Supervision (2004) as:

"the risk of direct or indirect loss resulting from inadequate or failed internal processes, people and systems or from external events. This definition includes legal risk, but excludes strategic and reputational risk."

This paper deals with the most elaborate approach for calculating the operational risk capital requirements, the loss distribution approach, which relies on internal simulations of potential loss distributions.

Several books have been published on operational risk quantification, such as McNeil et al (2005) and Panjer (2006). They stress the relevance of extreme value theory (EVT) in the loss distribution approach, and give an accurate overview of the mathematical methods currently available for this purpose. In addition, concrete studies on operational loss data have been performed over the past few years. We refer in particular to Moscadelli (2004), De Fontnouvelle et al (2004) and Dutta and Perry (2006).

In this paper we present an empirical study highlighting the challenges of quantifying operational losses, and some shortfalls of standard methodologies. Our results are based on real operational risk data, so we hope that this analysis will contribute to linking the purely theoretical properties of heavy-tailed distributions to the practical analysis of operational risk.

In the first section, we show that EVT provides us with appropriate tools to fit our data and model the heavy-tailedness that usually characterizes operational losses. We calculate capital charges at the enterprise and business line level. We investigate the consequences of using value-at-risk (VaR) as a risk measure and discuss the implications of dealing with heavy-tailed data. In particular, we examine the stability and accuracy of the capital charges as calculated following the Basel II requirements.

In the second section, we use copulas to aggregate the losses coming from different business lines. We show that the nature of the copula does not play a crucial role compared to the degree of heavy-tailedness of the data.

Our analysis is based on data collected from an individual bank database over a time window of four years, between 2002 and 2006, categorized in nine business lines and seven event types. The overall number of observations is 7,514.

\section{MODELING OPERATIONAL RISKS USING EXTREME VALUE THEORY}

In this part we model the losses that are part of our data set. They are first considered regardless of the business line they belong to, then every business line is examined separately. The aim is to show that EVT is needed to capture some stylized effects of the data, and to discuss the accuracy of standard risk measures. The model construction is detailed as well as the estimation procedure. 
FIGURE 1 Time series of aggregated data.

Aggregated losses (euros)

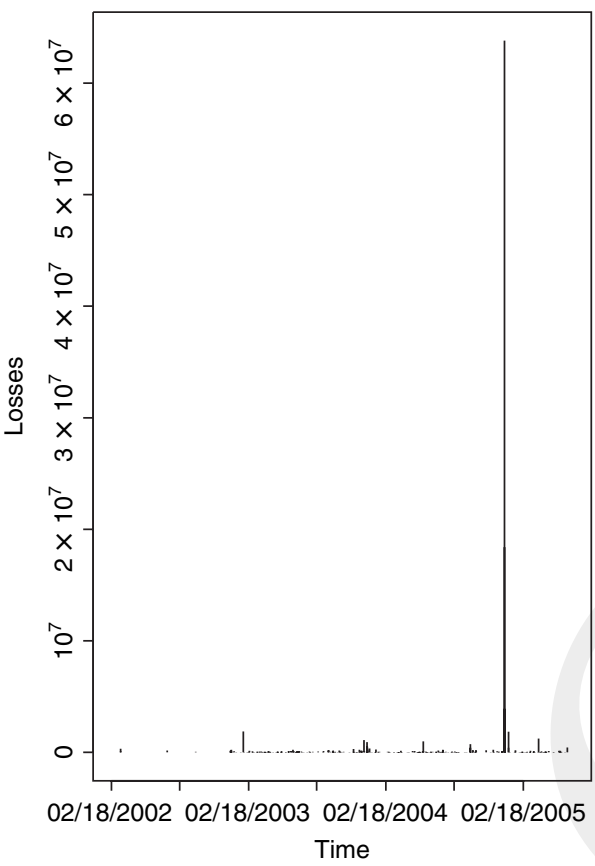

Zoom on the losses $<10^{6}$

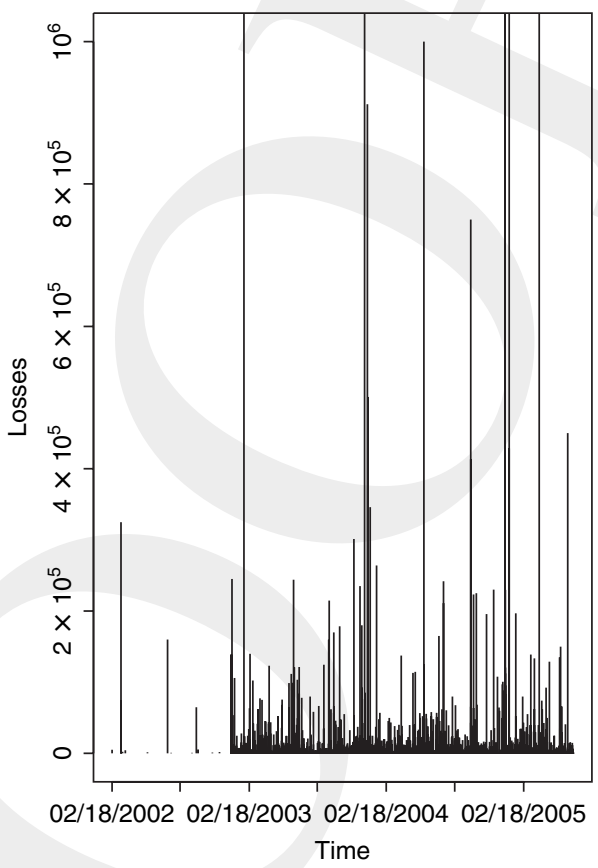

\subsection{Why use EVT?}

To start, let us perform a preliminary analysis of our data set using classical statistical tools. We assume here that our losses are independent and identically distributed (iid) realizations of a random variable with distribution function $F$.

Figure 1 shows the time series of losses, ordered by their time of discovery. The second graph is a zoom of the first graph, truncating losses to $10^{6}$.

We can observe some classical stylized effects that are in line with Embrechts et al (2003) and Chavez-Demoulin and Embrechts (2004). In particular, the graphs exhibit several extreme losses, incurring a high skewness (estimated equal to 64.17) and kurtosis (estimated equal to 114) of the loss distribution. Other effects include the non-stationarity of the data and a potential reporting bias, ${ }^{1}$ due to the fact that losses were usually not rigorously reported a few years ago. Thus, the overall data set used in this analysis appears to capture the usual trends that characterize operational risk.

We fit classical distributions to the data using the criteria of maximum likelihood, starting from light-tailed distributions (eg, Weibull), to medium-tailed distributions

\footnotetext{
${ }^{1}$ Frachot et al (2004a) evaluate the impact of the reporting bias on capital charges estimates.
} www.thejournalofoperationalrisk.com 
TABLE 1 Classical distributional fittings.

\begin{tabular}{lcccc}
\hline & $\mathbf{K S}$ & $\mathbf{A D}^{\mathbf{a}}$ & $\mathbf{C v}_{\mathbf{5} \%} \mathbf{K S}$ & $\mathbf{C v}_{\mathbf{5} \%} \mathbf{A D}$ \\
\hline Exponential & 0.43 & $+\infty$ & 0.007 & 0.20 \\
Weibull & 0.07 & $+\infty$ & 0.007 & 0.23 \\
Gamma & 0.07 & $+\infty$ & 0.007 & 0.21 \\
Lognormal & 0.015 & 3.78 & 0.007 & 0.23 \\
\hline
\end{tabular}

Kolmogorov-Smirnov is denoted as KS and Anderson-Darling is denoted as AD.

aBy construction, the $A D$ test yields infinite values when the theoretical distribution has a finite endpoint that is below that of the empirical distribution.

(eg, Gamma, exponential and lognormal). We adapt the Kolmogorov-Smirnov test (see Eadie et al (1971)) and the Anderson-Darling test (see Anderson and Darling (1952)) to measure the distance between the empirical and theoretical distribution functions only in the tail area, after the 93\% quantile. Table 1 reports the goodnessof-fit values as well as the critical values (denoted as $\mathrm{cv}$ in tables and figures), computed using Monte Carlo simulations.

All $p$-values are equal to zero. The lognormal distribution is the only model for which the Anderson-Darling test value is finite. However, it clearly does not reproduce the heavy-tailedness of the data. The failure of conventional distributions for our purpose is due to rare and extreme events, which are not captured by usual statistical tools.

\subsection{Analysis of the aggregated losses using EVT}

The key attraction of EVT is that it focuses on the analysis of the tail area of the distribution, providing appropriate methods for modeling extreme losses and their impact in insurance, finance and quantitative risk management. Thus, it has developed very quickly over the last decade. Specific conditions on the data are required to use EVT, which explains why it is still open to criticism, as discussed in Embrechts et al (2003, 2004). McNeil et al (2005) detail in Chapter 10 of their book the statistical methods used in EVT, applied to finance and insurance. The original mathematical theory is available in Embrechts et al (2002). The terminology used throughout this paper is the same as in McNeil et al (2005).

EVT provides an accurate model for exceedances over a high threshold by the means of the generalized Pareto distribution (GPD), defined as follows:

$$
G P D_{\xi, \beta}(x)= \begin{cases}1-\left(1+\frac{\xi x}{\beta}\right)^{-1 / \xi}, & \xi \neq 0 \\ 1-\exp \left(-\frac{x}{\beta}\right), & \xi=0\end{cases}
$$

The Journal of Operational Risk

Volume 4/Number 3, Fall 2009 
where $\beta>0, x \geq 0$ when $\xi \geq 0$ and $0 \leq x \leq(-\beta / \xi)$ when $\xi<0$. Parameters $\xi$ and $\beta$ are referred to, respectively, as the shape and scale parameters. Basically, $\xi$ is the key parameter of the distribution. It indeed reflects the level of heavy-tailedness of the data. We refer to McNeil et al (2005) for further explanations of the role and properties of parameters.

The main theorem of that approach has been developed by Balkema and de Haan (1974) and Pickands (1975). It states that under assumptions that are satisfied for most of the classical distributions, the distribution of the excess losses over a certain threshold converges towards a GPD when the threshold goes towards the right endpoint of the loss distribution. We refer to McNeil et al (2005, p. 277) for the exact formulation.

Intuitively, this means that the distribution of the excess losses over a threshold that is high enough can be approximated by a GPD. In light of this theorem, we choose a threshold and fit separately the body and the tail of the distribution. We use conventional inference to model the left part of the distribution and a GPD to model the tail.

\subsubsection{Tail calibration}

We use a mean-excess plot, as defined in McNeil et al (2005, p. 279), to assess the validity of modeling the tail by a power-law distribution such as the GPD. Figure 2 (see page 6) represents the mean excess function of the aggregated losses. The graph presents a clear upwards linearity, indicating that the data has a reasonable chance to be well modeled by a GPD.

Calibrating the tail of the distribution is equivalent to finding the optimal scale and shape parameters so that the corresponding GPD accurately approximates excess losses over the threshold chosen.

The Hill method is a well-known approach to get a rough estimate of the shape parameter. The Hill estimator $\hat{\xi_{k}}=\left(1 / \hat{\alpha_{k}}\right)$, as defined in Hill (1975) and detailed in McNeil et al (2005) and Koedijk et al (1990), has become a benchmark in the literature due to its easy implementation and asymptotic unbiasedness. It has been shown to be consistent with fat-tailed distributions (see Mason (1982)), and has been extensively studied for its practicalities. Furthermore, if the underlying loss distribution is Pareto, then the Hill estimator is the maximum likelihood estimate of the shape parameter. The primary weakness of this index lies in the need to determine the size of the tail a priori through the determination of the number of observations $k$ in the tail area. For certain types of functions including the GPD, a small $k$ helps decrease the bias because the power law is assumed to hold only in the extreme tail, but a large $k$ reduces variance - since more data points are used - and hence yields a better precision.

A Hill plot $\left\{\left(k, \hat{\alpha_{k}}\right)\right\}$ and the corresponding 0.90 Wald confidence interval for $\hat{\alpha_{k}}$ are drawn on Figure 3 (see page 7).

The graph suggests that the shape parameter lies between 0.65 and 1 , meaning that our data comes from a heavy-tailed distribution. This result is confirmed by using the 
FIGURE 2 Mean excess plot of the aggregated data.

03 selection. the choice of the threshold. model has finite mean or not.

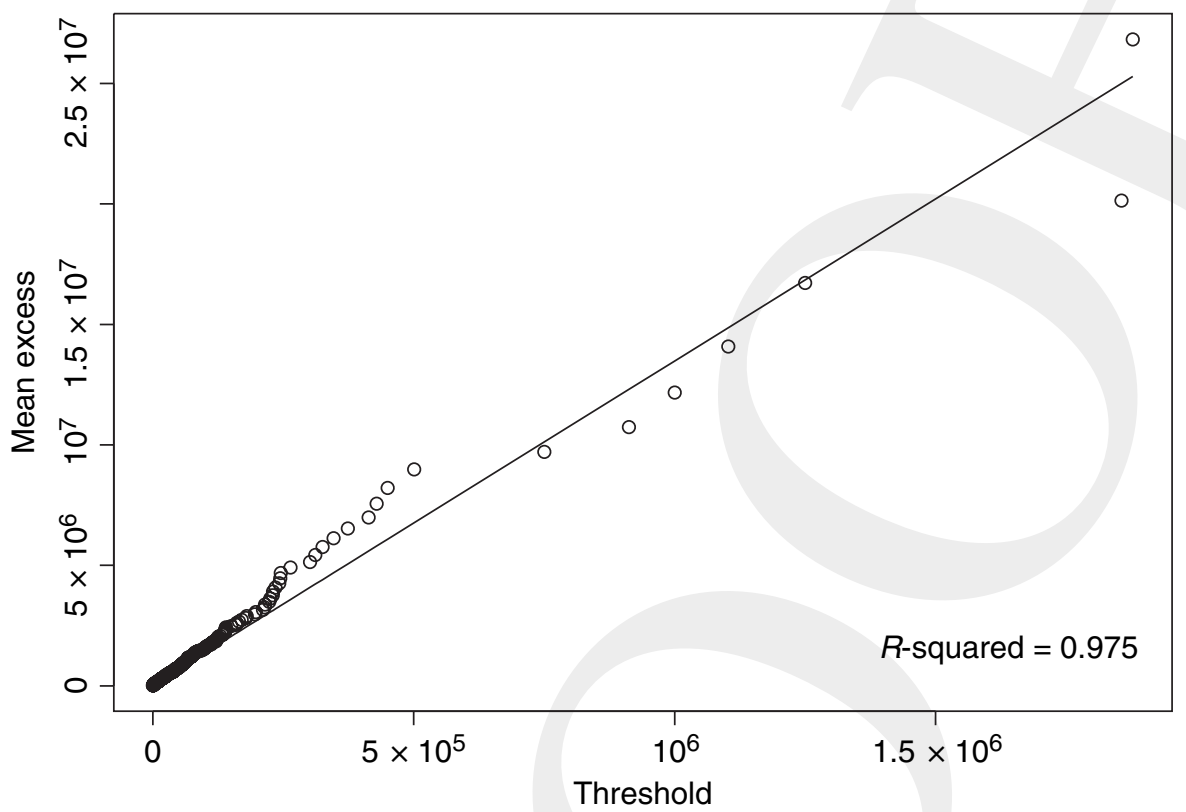

regression-based EVT technique proposed by Huisman et al (2001), which corrects the bias of the Hill estimator in small samples and minimizes the role of the threshold

The selection of the threshold is a key modeling aspect. Indeed, it should be large enough to satisfy the limit law condition. However, it has to leave enough observations to accurately estimate the severity of the tail. To evaluate the stability of the shape parameter, a shape plot, plotting the $\xi$ estimates across a variety of thresholds, is used. For details on that technique we refer to Embrechts et al (2002, p. 339). Figure 4 (see page 8 ) shows that the shape parameter is not very sensitive to

We fix the threshold at the $90 \%$ percentile and model the excess data with a GPD. The parameters are calibrated by maximizing the likelihood function. The GPD scale and shape estimates are, respectively, $\hat{\beta}=10691.28$ and $\hat{\xi}=0.89$. The 0.95 confidence interval $[0.75 ; 1.05]$ for the shape parameter is obtained through a bootstrapping procedure. This interval outlines the uncertainty about whether the

Figure 5 (see page 9) displays two graphical plots illustrating the quality of the fitting: the first one represents the tail of the empirical distribution versus that of the fitted GPD, and the second one is a quantile-quantile-plot of the excesses. 
FIGURE 3 Hill plot of the aggregated losses and 0.95 confidence interval.

Hill plot

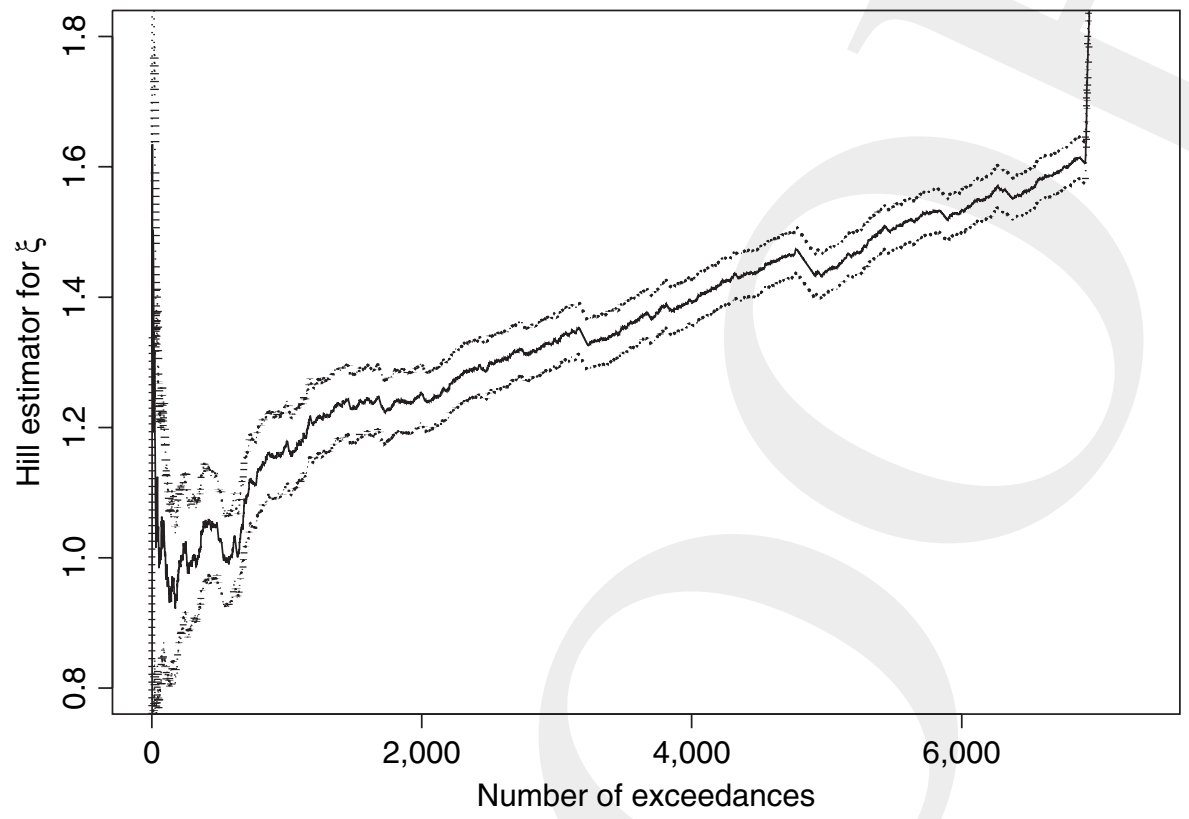

Following Moscadelli (2004), a severity VaR performance analysis is performed to compare the different levels of accuracy of the GPD versus the lognormal distribution in representing the highest percentiles of the data. The expected and the estimated number of violations are compared for several given levels of confidence of the VaR. Table 2 (see page 10) provides the results of the tests.

The high number of violations when the tail is modeled using a lognormal distribution indicates a large underestimation of the large losses. The calibrated GPD model gives quantiles of the distribution that are much closer to the empirical quantiles and therefore the model accurately reflects extreme events.

\subsubsection{Final model for the loss severities}

Losses below the threshold are modeled by a lognormal distribution and excesses by a GPD as detailed above. In the following, this model is referred to as lognormal GPD. It is constructed as follows.

Let us call $F_{1}$ the distribution before the threshold (lognormal), $F_{2}$ the distribution of the excesses (GPD) and $F$ the distribution of the whole data set. Then, with a 
FIGURE 4 Shape plot of the aggregated data.

Threshold

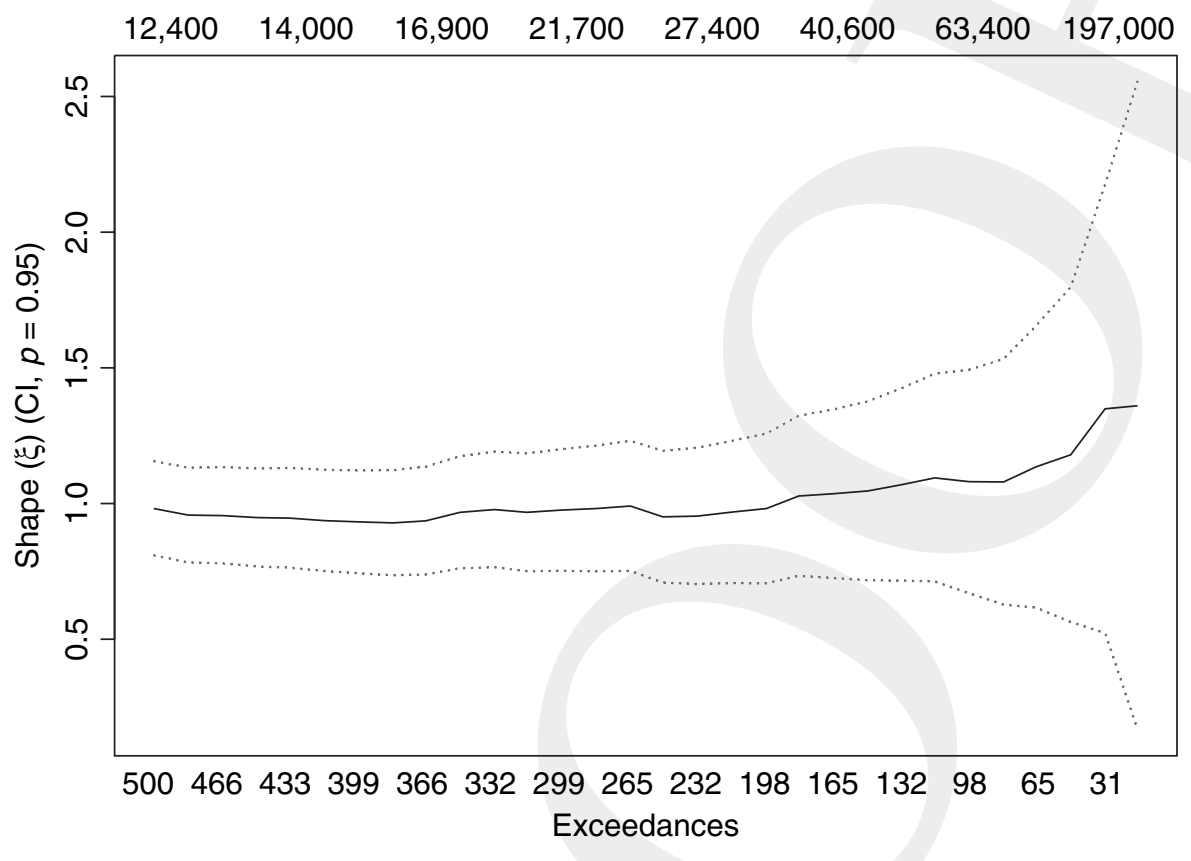

threshold fixed at the $90 \%$ percentile, we have:

$$
\begin{aligned}
\forall x, \quad F(x) & =P(X \leq x) \\
& =P(X \leq x \mid X \leq u) \cdot P(X \leq u)+P(X \leq x \mid X>u) \cdot P(X>u)
\end{aligned}
$$

Hence:

$$
\forall x \leq u, \quad F(x)=F_{1}(x) \cdot 0.90+0
$$

and:

$$
\forall x>u, \quad F(x)=1 \cdot 0.90+F_{2}(x-u) \cdot 0.10 .
$$

Figure 6 (see page 11) shows a comparison of the empirical cumulative distribution function (cdf) to the lognormal cdf (graph 1) and the lognormal GPD cdf (graph 2). The latter clearly provides a better fit. Table $3^{2}$ (see page 12 ) provides the goodnessof-fit test values of the lognormal GPD model and confirms that using EVT significantly improves the results.

\footnotetext{
${ }^{2}$ To be compared to the results obtained in Table 1 .
}

The Journal of Operational Risk 
FIGURE 5 Tail plot and quantile-quantile-plot of the excesses.
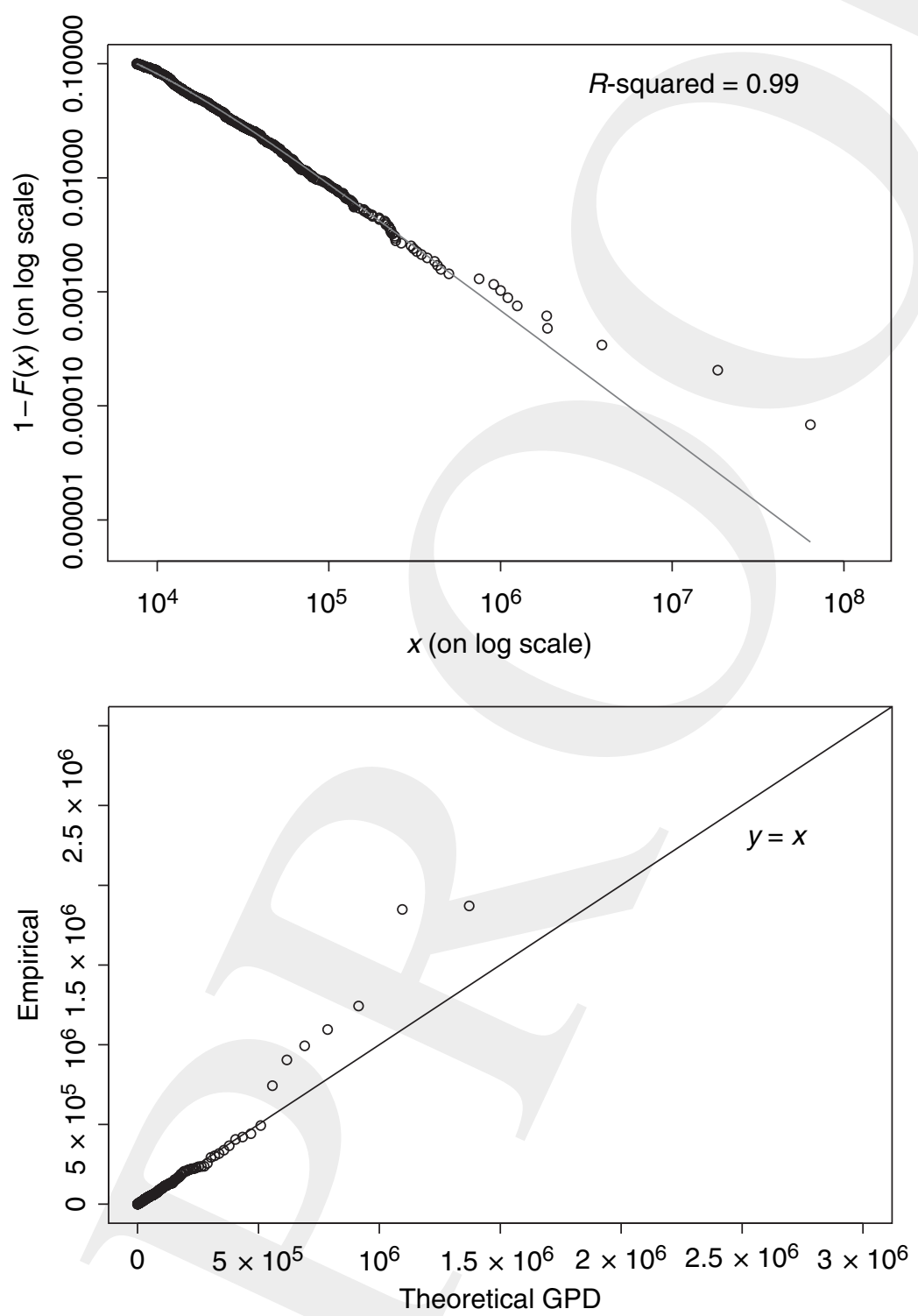
TABLE 2 Number of violations for different distributions.

\begin{tabular}{lccc}
\hline & \multicolumn{3}{c}{ Number of violations } \\
\cline { 2 - 4 } Level of confidence & Empirical & GPD & Lognormal \\
\hline 0.950 & 36.85 & 37 & 48 \\
0.975 & 18.42 & 18 & 35 \\
0.990 & 7.37 & 10 & 20 \\
0.995 & 3.68 & 5 & 16 \\
\hline
\end{tabular}

\subsubsection{Calculation of the capital charges for aggregated losses}

Let the total claim amount be equal to $S_{N}=\sum_{i<N} X_{i}$, where $N$, the number of claims, and $X_{i}$, the loss amounts, are assumed to be independent. We estimate $S_{N}$ with Monte Carlo simulations and model the data frequency by a homogeneous Poisson process with daily intensity 6.08 . The claims' severities follow the model previously described. ${ }^{3}$ The time horizon is chosen to be equal to one year, following the Basel II requirements. Results of the simulation for the VaR are reported (in millions ${ }^{4}$ ) in Table 4 (see page 12).

These results are coherent with the yearly quantiles of the data. Nevertheless, the capital charges estimate grows very quickly with the quantile chosen: the $0.999 \mathrm{VaR}$ is more than six times larger than the $0.99 \mathrm{VaR}$. This motivates a sensitivity analysis with respect to the key parameters.

\subsubsection{Sensitivity analysis of the capital charges}

In this section we study the sensitivity of the VaR to the shape parameter, the level of confidence and the frequency model.

- Sensitivity to the shape parameter. The Hill plot and confidence interval for the shape parameter $\xi$ have suggested a fairly strong uncertainty. Figure 7 (see page 12) illustrates the impact of the shape parameter on the VaR, for different levels of confidence.

The $99.9 \% \mathrm{VaR}$ is very unstable and requires a higher number of simulations. In a reasonable amount of time, it cannot be relied upon for a precise estimate of capital charges. Furthermore, the larger the shape parameter, the less stable the VaR, especially for $\xi>1$. This is in line with Neslehova et al (2006), who show that, in an infinite-mean model, the VaR grows with the tail index parameter at an exponential rate. This is one reason why the heavy-tailedness of our data represents a challenge to precisely calculate the capital charges.

\footnotetext{
${ }^{3}$ The losses that are below the threshold are modeled using a lognormal distribution and the excesses over the threshold by a GPD.

${ }^{4}$ Results are rounded due to the imprecision inherent in the use of Monte Carlo simulations. 
(a)

(b)$$
\text { . }
$$$$
\text { . }
$$
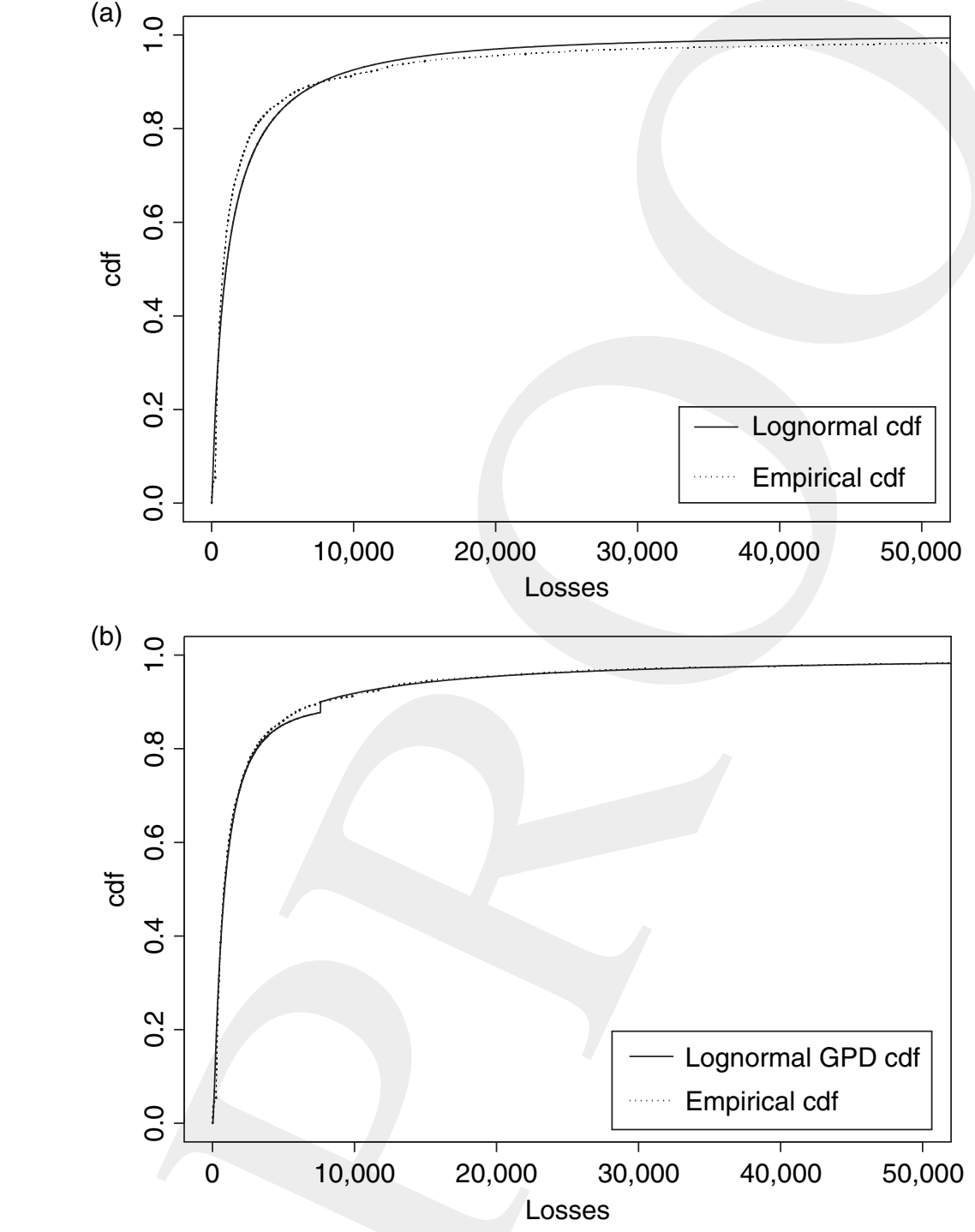

FIGURE 6 Theoretical versus empirical distributions for (a) a lognormal model and (b) a lognormal GPD model. 
TABLE 3 Goodness-of-fit test values for the lognormal-GPD approach.

\begin{tabular}{cccc}
\hline $\mathbf{K S}$ & $\mathbf{A D}$ & $\mathbf{C v}_{\mathbf{5} \%} \mathbf{K S}$ & $\mathbf{c v}_{\mathbf{5} \%} \mathbf{A D}$ \\
\hline 0.003 & 0.026 & 0.005 & 0.13 \\
\hline Kolmogorov-Smirnov is denoted & as & $\mathrm{KS}$ and \\
Anderson-Darling is denoted as AD.
\end{tabular}

TABLE 4 One-year VaR.

\begin{tabular}{ccccc}
\hline & \multicolumn{4}{c}{ Confidence level } \\
\cline { 2 - 5 } & $\mathbf{0 . 9 0}$ & $\mathbf{0 . 9 5}$ & $\mathbf{0 . 9 9}$ & $\mathbf{0 . 9 9 9}$ \\
\hline $\mathrm{VaR}_{\text {conf\% }}$ & 26 & 36 & 104 & 639 \\
\hline
\end{tabular}

FIGURE 7 One-year VaR versus shape parameter.

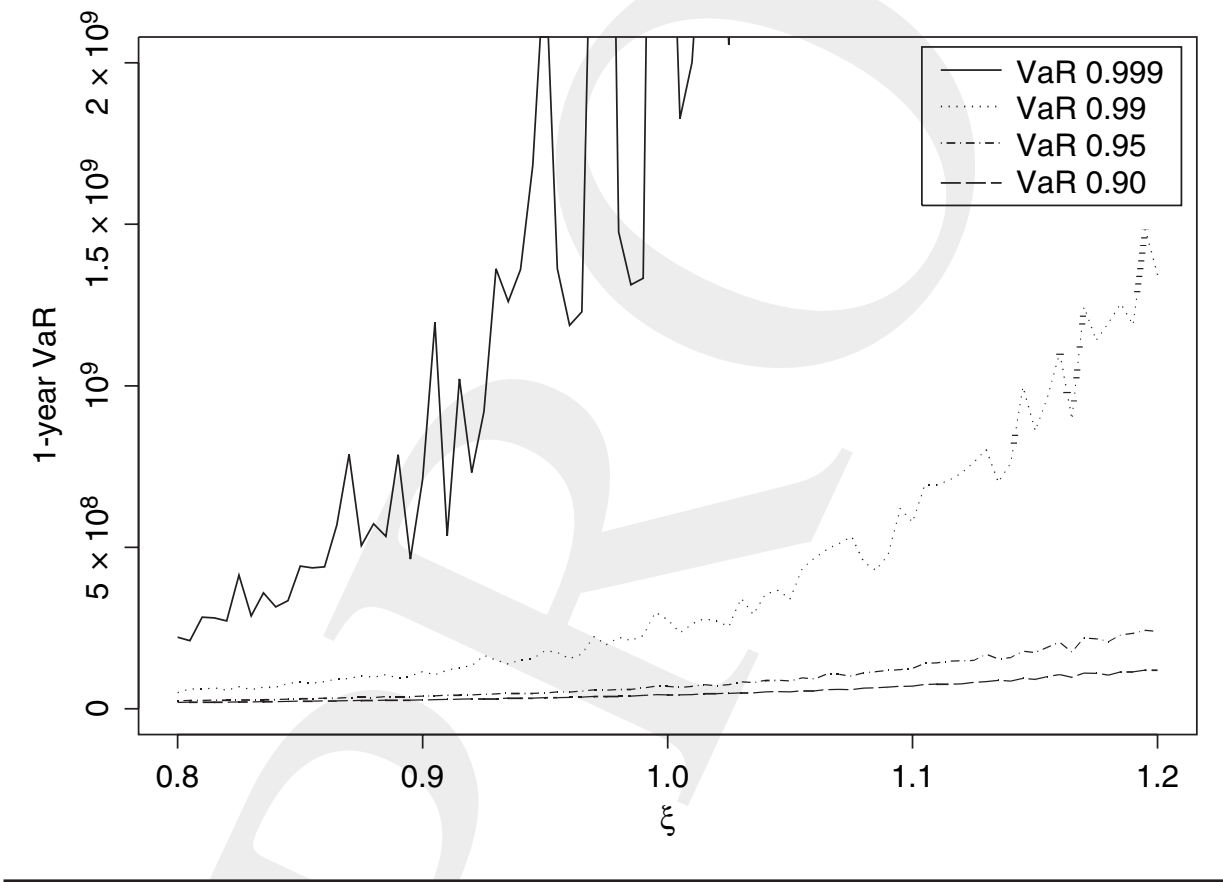

- Sensitivity to the level of confidence. The Basel II Accord specifies that capital charges must be calculated at the 0.999 quantile of the loss distribution. Figure 8 (see page 13) illustrates the relationship between the one-year VaR and the quantile it is computed at. 
FIGURE 8 One-year VaR versus confidence level.

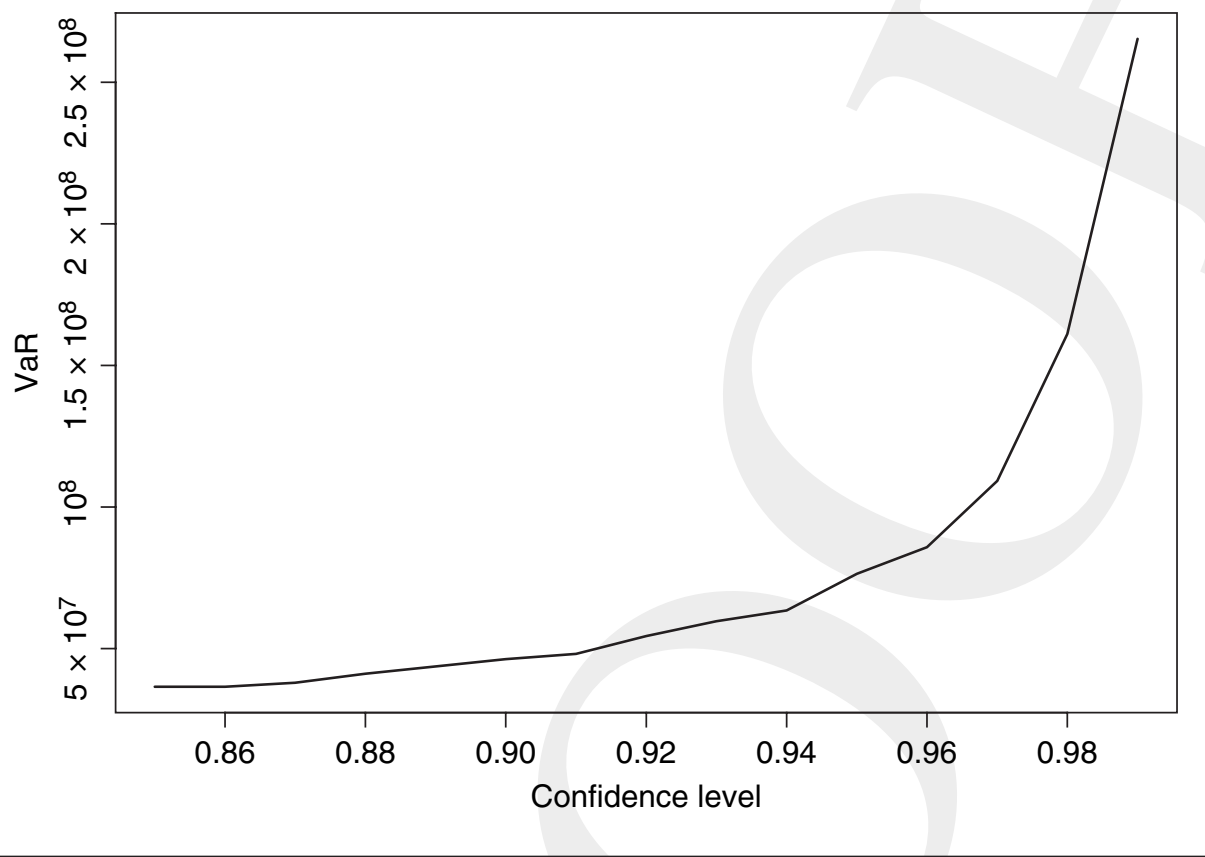

The graph is quite stable between the $0.85 \%$ and $0.92 \%$ quantiles, then it increases exponentially. So the VaR is not very robust with respect to the confidence level.

- Importance of the frequency model. We took a Poisson process to simulate the loss frequency. This choice does not reflect the over-dispersion of our data (see also Evans et al (2007)). The negative binomial distribution provides a way to solve this issue. However, Table 5 (see page 14) shows that the differences in the resulting capital charges are quite small (around 5\%) before the 0.999 level of confidence. After this level, the VaR is higher than when using the Poisson model, which already seems to overestimate the capital charges. This motivates the use of the Poisson model in the rest of this study.

\subsection{Analysis of the losses by business line using EVT}

The analysis of the entire data set highlights some general properties of the data, which are particular to operational losses. However, we also conduct an analysis at the business line level, since it is likely to outline inhomogeneities from one business line to another. Owing to a lack of data, we restrict ourselves to the business lines (BL) for which we have more than 250 data points: BL1, BL3, BL6, BL7 and 
TABLE 5 Capital charges using the negative binomial distribution for frequencies.

\begin{tabular}{lcccc}
\hline & \multicolumn{4}{c}{ Confidence level } \\
\cline { 2 - 5 } & $\mathbf{0 . 9 0}$ & $\mathbf{0 . 9 5}$ & $\mathbf{0 . 9 9}$ & $\mathbf{0 . 9 9 9}$ \\
\hline VaR negative binomial & 28 & 38 & 99 & 724 \\
VaR negative binomial/Poisson & $\times 1.04$ & $\times 1.05$ & $\times 0.95$ & $\times 1.13$ \\
\hline
\end{tabular}

TABLE 6 Final models for each business line.

\begin{tabular}{llccc}
\hline Business line & Distribution & Threshold & $\hat{\boldsymbol{\xi}}$ & Confidence interval \\
\hline BL1 & Lognormal GPD & 0.85 & 0.87 & {$[0.55 ; 1.23]$} \\
BL3 & Lognormal GPD & 0.65 & 1.70 & {$[1.13 ; 2.25]$} \\
BL6 & GPD & - & 0.85 & {$[0.77 ; 1.01]$} \\
BL7 & Lognormal GPD & 0.90 & 1.05 & {$[0.85 ; 1.20]$} \\
BL9 & Lognormal GPD & 0.75 & 0.37 & {$[0.13 ; 0.60]$} \\
\hline
\end{tabular}

TABLE 7 VaR of the severity distribution of the business lines modeled with GPD distributions.

\begin{tabular}{lcrr}
\hline & \multicolumn{3}{c}{ Confidence level } \\
\cline { 2 - 4 } Business line & $\mathbf{0 . 9 5}$ & \multicolumn{1}{c}{$\mathbf{0 . 9 9}$} & \multicolumn{1}{c}{$\mathbf{0 . 9 9 9}$} \\
\hline BL1 & 11,396 & 47,549 & 330,467 \\
BL3 & 45,309 & 733,620 & $39,735,356$ \\
BL6 & 23,104 & 127,463 & $1,408,266$ \\
BL7 & 10,297 & 43,150 & 321,986 \\
BL9 & 50,973 & 118,403 & 322,985 \\
\hline
\end{tabular}

BL9. We now assume that losses that belong to $\mathrm{BL}_{i}$ are iid realizations of $i$ random variables with distributions $F_{i}$.

\subsubsection{Final individual models}

Based on the results of conventional inference and EVT, and following the approach described in Section 2.2, the models reported in Table 6 are inferred.

Shape parameters indicate that BL9 is much lighter-tailed than the other business lines; BL1, BL6 and BL7 are quite heavy-tailed with shape parameters very close to one, and BL3 is the heaviest-tailed BL, with a shape parameter close to two.

\subsubsection{Analysis of the tail severity}

Using the properties of the GPD described in McNeil et al (2005, p. 283), we calculate the VaR of the business lines' severities, to give an idea of the magnitude of the tail. Table 7 reports the results.

The Journal of Operational Risk

Volume 4/Number 3, Fall 2009 
TABLE 8 One-year VaR for each business line.

\begin{tabular}{lrrrr}
\hline & \multicolumn{4}{c}{ Confidence level } \\
\cline { 2 - 5 } Business line & \multicolumn{1}{c}{$\mathbf{0 . 9 0}$} & \multicolumn{1}{c}{$\mathbf{0 . 9 5}$} & \multicolumn{1}{c}{$\mathbf{0 . 9 9}$} & \multicolumn{1}{c}{$\mathbf{0 . 9 9 9}$} \\
\hline BL1 & 1,846 & 2,696 & 7,963 & 48,430 \\
BL3 & 43,865 & 138,513 & $2,134,864$ & $107,031,623$ \\
BL6 & 3,905 & 6,197 & 23,118 & 200,134 \\
BL7 & 25,546 & 42,798 & 184,316 & $1,967,351$ \\
BL9 & 2,691 & 2,897 & 3,396 & 4,486 \\
\hline
\end{tabular}

TABLE 9 Confidence intervals of the VaR for BL1.

\begin{tabular}{cc}
\hline Confidence level & Confidence interval \\
\hline 0.90 & {$[2,073 ; 2,220]$} \\
0.95 & {$[3,120 ; 3,430]$} \\
0.99 & {$[9,347 ; 12,225]$} \\
0.999 & {$[38,325 ; 121,738]$} \\
\hline
\end{tabular}

Owing to the heavy-tailedness of the data, the VaR grows very rapidly with the quantile it is calculated at. Furthermore, this table confirms the importance of BL3 regarding the amount of money the bank has to put aside. At the $99.9 \%$ percentile, the VaR corresponding to BL3 is 17 times larger than the sum of all the VaRs of other business lines modeled by GPD distribution. The biggest losses in BL3 therefore have a fundamental impact on the overall capital charges.

This is in line with the concept of subexponentiality, ${ }^{5}$ referred to in Embrechts and Puccetti (2006) as the "one loss causes ruin problem". This issue arises for heavytailed distributions including the GPD and lognormal distribution. For instance, for a tail index close to one, which is our case for BL1 and BL7, $0.1 \%$ of the losses produce $95 \%$ of the total losses. This means that one single loss can have a tremendous impact on the company's healthiness.

\subsubsection{Capital charges at the business line level}

Capital charges are computed and reported (in thousands) in Table 8, with regards to the confidence level of the VaR.

The confidence intervals for the VaR calculation are very large for the highest percentiles, as illustrated by Table 9 for BL1. This means that the VaR cannot be accurately determined at a high level of precision given the data we have.

\footnotetext{
${ }^{5}$ See Mandelbrot and Hudson (2004, p. 232), for a discussion of the consequences of subexponentiality. 
TABLE 10 Coherence of the VaR.

\begin{tabular}{lcc}
\hline & $\operatorname{VaR}\left(\sum_{\left.\boldsymbol{i}=\mathbf{1 , 3 , 7 , \boldsymbol { g }} \boldsymbol{B} \boldsymbol{L}_{\boldsymbol{i}}\right)}\right.$ & $\sum_{\boldsymbol{i}=\mathbf{1 , 3 , 7 , \boldsymbol { 9 }}\left(\operatorname{VaR} \boldsymbol{B} \boldsymbol{L}_{\boldsymbol{i}}\right)}$ \\
\hline 0.90 & 87 & 74 \\
0.95 & 214 & 187 \\
0.99 & 2,461 & 2,331 \\
0.999 & 126,007 & 109,052 \\
\hline
\end{tabular}

Furthermore, total capital charges mainly reflect the big losses that have occurred in BL3. Very few events drive the dynamics of the whole system, as described in Section 2.3.2.

Let us point out that the VaR for BL3 is higher than the global VaR for aggregated losses, as calculated in Table 4 . We explain that fact by the uncertainty inherent to both approaches: modeling the whole data set does not take into account the inhomogeneity of the data, and considering every single business line makes the data sets much smaller, hence introducing more uncertainty into the calibration.

\subsubsection{Accuracy of the capital charges by business line}

A fundamental question is raised at this level of the study: are our calculations of VaR accurate to determine the capital requirements? It is well known that the VaR does not satisfy the subadditivity axiom described in Delbaen (2002), and is therefore a non-coherent risk measure. The comparison of the sum of the VaR for all business lines (in millions), modeled with a lognormal GPD distribution, to the VaR of their sum is summarized in Table 10. Let us point out that BL3, which satisfies $\xi>1$, is part of the current calculation.

The results show that the VaR is superadditive, meaning that care has to be taken when choosing it as the risk measure to compute capital charges when models have infinite mean as is the case for BL3. Following Neslehova et al (2006), these kinds of models cause serious problems regarding diversification. Indeed, they yield higher capital charges for uncorrelated losses than for correlated losses, which goes against the diversification principle.

We make the same calculations without BL3, ie, only with business lines that have shape parameters smaller than or around one (BL7). We get the results reported in Table 11 (see page 17).

The differences between the sum of the VaR and the VaR of the sum are much less significant here. Therefore, results suggest that distributions satisfying $\xi \leq 1$ allow us to approximate the capital charges by the sum of the business lines' individual VaR with more realism. The same calculations when removing BL7 confirm this result. The heavier-tailed the data, the more dangerous and unrealistic the computation of the capital charges. 
TABLE 11 Coherence of the VaR for less heavy-tailed distributions.

\begin{tabular}{lcc}
\hline & $\operatorname{VaR}\left(\sum_{i=1,7,9} \boldsymbol{B} L_{\boldsymbol{i}}\right)$ & $\sum_{\boldsymbol{i}=\mathbf{1 , 7 , 9}}\left(\operatorname{VaR} \boldsymbol{B} \boldsymbol{L}_{\boldsymbol{i}}\right)$ \\
\hline 0.90 & 30 & 30 \\
0.95 & 47 & 48 \\
0.99 & 196 & 195 \\
0.999 & 1,922 & 2,020 \\
\hline
\end{tabular}

In theory, Proposition 4.1 of Degen et al (2007) states that, when $\xi<1, \mathrm{VaR}_{\alpha}$ is subadditive for $\alpha$ sufficiently large. This has critical implications in terms of risk management. Indeed, we have shown that VaR was neither stable nor reliable at very high quantiles. In practice, this is often dealt with by calculating it at smaller quantiles, for instance 0.90, and scaling it up. Our study shows that this is not accurate since VaR might get superadditive and lead to underestimated capital charges if infinite-mean distributions are involved.

\subsubsection{Concluding remarks on the application of $\mathrm{VaR}$ to heavy-tailed data}

What should be remembered from this is that, in the case of extremely heavy-tailed loss distributions, standard economic thinking about diversification and VaR are inappropriate. The analysis of our data is in line with the literature and emphasizes the pitfalls of using VaR as a risk measure. However, Delbaen (2002) shows that any coherent risk measure $\rho$ that is dependent of the distribution function of the risks and for which $\rho \geq V a R_{\alpha}$ has to satisfy $\rho \geq E S_{\alpha}$, where $E S$, the expected shortfall, requires the existence of the first moment (ie, $\xi<1$ ). This means that expected shortfall is the smallest coherent risk measure to be greater than VaR, and hence that there does not exist any coherent risk measure larger than VaR that yields finite capital charges if the losses are GPD with $\xi>1$.

\section{MODELING OF THE DEPENDENCY STRUCTURE OF OPERATIONAL RISKS WITH COPULAS}

Calculating the minimum capital requirements as the sum of the VaR over the different business lines assumes a perfect dependence between them. However, Frachot et al (2004b) argue on the one hand that operational risk models cannot, by construction, show high levels of correlation between losses from different business lines, suggesting that the capital charges are highly overestimated. On the other hand, Embrechts and Puccetti (2006) point out that, when VaR is not subadditive, dependence can lead to an underestimation of the capital requirements. In this section, we see how copulas can be used to describe dependencies and we analyze the variations of capital they lead to. 
Copulas express dependence on a quantile scale and facilitate a bottom-up approach for building multivariate models. ${ }^{6}$ Many successful applications have been recently developed, particularly in actuarial science, survival analysis and hydrology. In finance, the methodology is extensively studied in the books of Cherubini et al (2004) and McNeil et al (2005).

\subsection{Preliminary analysis of the dependence structure}

Coefficients of tail dependence and rank correlations are used to detect dependence between the different business lines. They measure pairwise extremal dependence and only depend on the copula of a pair of random variables. A formal definition as well as further explanations on the intuition behind can be found in McNeil et al (2005, pp. 206-209).

To evaluate these coefficients, we calculate monthly total losses for each business line and assume that the dependence structure on a monthly time horizon is identical to that on a yearly basis. Empirical pairwise coefficients of upper tail dependence are around 0.5 between BL1, BL6, BL7 and BL9. All pairs of business lines present lower tail dependence, which is not surprising considering the large number of small losses in the database.

The computation of Spearman and Kendall rank correlation matrices, $\rho_{S / \tau}\left(B L_{i}, B L_{j}\right)$, for the five business lines that we considered in the last section, clearly shows that none of the pairs of business lines are perfectly correlated with each other. This goes along with Frachot et al (2004b). The rank correlations are in most cases quite weak, except for BL1 and BL9. As in Genest and Favre (2007), we performed a test of independence based on Kendall's $\tau$. The hypothesis $H_{0}$ that two business lines are independent is only rejected for BL1 and BL9.

Many copulas are compatible with this dependence structure. However, very few of them can be used in a $n$-dimensional framework with $n>2$. We will now examine the capital charges resulting from the most standard copulas.

\subsection{Copula modeling}

First, we use meta-Gaussian and meta- $t$ copulas, taking the distributions calibrated in the first part as marginal distribution functions of the yearly losses. Parameters are fitted using the maximum likelihood approach, which requires the existence of the density of the copula. The pseudo-likelihood $\sum_{t=1}^{n} \ln c\left(\widehat{\mathbf{U}}_{\mathbf{t}}\right)$, where $\widehat{\mathbf{U}}_{\mathbf{t}}$ is the vector of pseudo-observations, is maximized, then we perform Monte Carlo simulations to calculate the capital charges. We use the procedure described in Clemente and Romano (2003).

\footnotetext{
${ }^{6}$ The copula approach provides a way to describe the joint distribution of a random vector of risk factors through the individual behaviors of each of the risk factors and the dependence structure that links them.
} 
TABLE 12 Variation of capital charges when using a meta-Gaussian copula.

\begin{tabular}{lcccr}
\hline & \multicolumn{4}{c}{ Confidence level } \\
\cline { 2 - 5 } & $\mathbf{0 . 9 0}$ & $\mathbf{0 . 9 5}$ & $\mathbf{0 . 9 9}$ & \multicolumn{1}{c}{$\mathbf{0 . 9 9 9}$} \\
\hline VaR comonotonicity & 65 & 163 & 2,076 & 98,669 \\
VaRp meta-Gaussian copula & 72 & 172 & 2,023 & 103,805 \\
VaR $/ d_{\text {meta-Gaussian copula }}$ & $\mathbf{7 2}$ & 172 & $\mathbf{2 , 1 4 5}$ & $\mathbf{9 7 , 2 1 7}$ \\
VaR meta-Student copula & 69 & 171 & $\mathbf{2 , 1 3 3}$ & 126,914 \\
\hline
\end{tabular}

TABLE 13 Variation of capital charges when excluding BL3.

\begin{tabular}{lcccc}
\hline & \multicolumn{4}{c}{ Confidence level } \\
\cline { 2 - 5 } & $\mathbf{0 . 9 0}$ & $\mathbf{0 . 9 5}$ & $\mathbf{0 . 9 9}$ & $\mathbf{0 . 9 9 9}$ \\
\hline VaR comonotonicity & 24 & 35 & 110 & 868 \\
VaR meta-Gaussian copula & 24 & 34 & 103 & 742 \\
\hline
\end{tabular}

The adjusted capital charges are compared to the sum of the capital charges over business lines - comonotonicity case - and Table 12 presents the VaR (in millions) at different levels of confidence, and in the case where all business lines would be independent.

The meta-Gaussian and meta- $t$ copula yield capital charges that are in most cases a little bit higher than if the business lines were comonotonic, ie, perfectly correlated. The difference is around 5\%. This means that decreasing correlation between business lines in the model by using copulas increases the capital charges. Again, this violates the principle of risk diversification and is due to the fact that VaR is superadditive for heavy-tailed data. Another illustration of that phenomenon is presented in the third row of the table, which reports the value of the VaR if the data were independent. Until the 0.999 percentile, VaR is higher in the independent case than in the comonotonic case. This is in line with Böcker and Klüppelberg (2006) (see Figure 3.13 therein) and proves, once more, that risk management in the case of heavy-tailed data can be problematic.

We evaluate the impact of the heaviest-tailed business line, BL3, by repeating the same procedure without including it in the computation. The results (in millions) are reported in Table 13.

Ignoring BL3, using copulas, and therefore decreasing the correlation between business lines in the model, allows a decrease of the capital charges. The gain increases with the percentile and reaches $14 \%$ for the 0.999 level of confidence. This confirms that the heavy-tailedness of BL3 was at the origin of the increase of the VaR when using copulas.

The Gaussian and $t$-copulas are copulas of elliptical distributions. This implies, as pointed out in Mikosch (2006), that all the realizations of the loss variables are given 
TABLE 14 Variation of capital charges with Frank and Cook-Johnson copulas.

\begin{tabular}{lcccc}
\hline & \multicolumn{4}{c}{ Confidence level } \\
\cline { 2 - 5 } & $\mathbf{0 . 9 0}$ & $\mathbf{0 . 9 5}$ & $\mathbf{0 . 9 9}$ & $\mathbf{0 . 9 9 9}$ \\
\hline Without copula & 65 & 163 & 2,076 & $\mathbf{9 8 , 6 6 9}$ \\
Frank copula & $\mathbf{7 6}$ & 180 & $\mathbf{2 , 1 1 9}$ & $\mathbf{8 7 , 4 3 8}$ \\
Cook-Johnson copula & 68 & 177 & 2,131 & 92,789 \\
\hline
\end{tabular}

the same importance in the statistical exercise. This goes against the basic principles of risk management, which gives more weight to the highest and hence most dangerous losses. Furthermore, the dependence structure of elliptical distributions is mainly determined by the covariance matrices. Embrechts et al (2001b) argue that it is not an accurate tool for risk modeling purposes.

Archimedean copulas allow for a greater variety of dependence structures. Embrechts et al (2001a) give a comprehensive overview of their mathematical properties. To fit the parameters using maximum likelihood, we use the close form expressions provided in Savu and Trede (2008). We calculate the capital charges resulting from Frank and Cook-Johnson copulas. We do not fit the Gumbel copula since it is an extreme-value copula, and hence, as explained in Mikosch (2006), it is not relevant for the structure of our data.

Table 14 shows that there is not much difference in the capital charges given by the two copulas. They both yield results that are very close to that of the meta-Gaussian copula, and do not reduce the capital charges for most of the percentiles.

If we exclude BL3, the capital charges remain unchanged for the 0.90 and 0.95 percentiles, and then are reduced by 20 to $30 \%$. So, in general, using copulas seems to reduce capital charges at the highest percentiles, but increases them for lower quantiles.

More flexible Archimedean copulas have been developed, allowing for more than one parameter, and hence for a better flexibility in the modeling of the dependence structure. In particular, a very promising alternative to standard copulas has been proposed by Joe (1993). He presents three types of nested Archimedean copulas: the fully, partially and hierarchical nested Archimedean copulas as well as the pair copula construction. We refer to the paper of Berg and Aas (2007) for further explanations on the benefits of such multidimensional copula constructions. Having more parameters requires much more data than the amount we have at our disposal, but might be worth exploring when more data points will be available.

\subsection{Evaluating the accuracy of a copula}

As is noticed by Mikosch (2006), it is difficult to see which copula should be used for a given set of data in more than two dimensions. Following Berg and Aas (2007, Section 4.1, p. 15), we choose the goodness-of-fit test suggested by Genest and 
TABLE 15 Goodness-of-fit (GoF) test values for the choice of the copula.

\begin{tabular}{lcccc}
\hline & Gaussian & $\boldsymbol{t}$ & Frank & Cook-Johnson \\
\hline GoF value & 0.027 & 0.025 & 0.107 & 0.832 \\
Cv $_{95 \%}$ & 0.083 & 0.131 & 0.218 & 0.414 \\
\hline
\end{tabular}

Rémillard (2004), which is based on the Euclidean distance between the hypothetical and empirical copula distributions, applied to the pseudo-observations.

Large values of the statistic $S$ lead to the rejection of the null hypothesis. Table 15 reports the test values and $95 \%$ critical values for the different copulas considered above.

The $t$-copula is the only copula we considered that has more than one parameter. This can explain why it yields the best test results.

\subsection{Concluding remarks on the modeling of the dependencies using copulas}

Using copulas to model the dependence structure allows for better realism but does not provide a solution to the calculation of capital charges when the model involves GPDs with $\xi>1$. Indeed, the superadditivity of the VaR prevents capital charges from decreasing as would be expected when the correlation between business lines in the model is decreased. Our results are in line with the existing literature and show that the standard copula approach yields results that contradict standard economic thinking about diversification.

\section{CONCLUSION}

Several issues have been empirically highlighted in this study. First, we have shown that heavy-tailed data sets are hard to model, and thus require much caution when interpreting the resulting capital charges. In the presence of infinite-mean models, a few losses in a business line may have a huge impact at the enterprise level and may drastically increase the capital charges. This leads to an obvious overestimation of the charges and to a lack of stability growing with the level of confidence of the VaR. We have emphasized the sensitivity of the overall analysis to the shape parameter of the distribution when the excesses are GPD, as well as that to the level of confidence. Furthermore, we have shown that, for heavy-tailed distributions, summing the VaR over business lines as recommended in the Basel II agreements is likely to lead to a underestimation of the capital charges due to the superadditivity of the VaR.

By modeling the dependence structure of the data using copulas, we have shown that infinite-mean models lead to an increase of the capital charges that goes against the diversification principle. The nature of the copula does not have a significant impact on the overall capital charges compared to that of the parameters previously 
mentioned. We have emphasized some theoretical limitations of the copulas considered, and introduced more sophisticated copulas allowing for a larger flexibility.

Finally, we think that the key message of this study is that heavy-tailed distributions with shape parameters bigger than one are the source of many theoretical problems including a high impact on the overall capital charges, instability and high uncertainty of the results, and incoherence of the VaR leading to a possible misestimation of the capital charges. Therefore, the shortfalls of the current modeling techniques need to be kept in mind. However, this is not a lost cause, and the studies on operational risk keep uncovering parts of the iceberg.

\section{REFERENCES}

Anderson, T. W., and Darling, D. A. (1952). Asymptotic theory of certain goodness of fit criteria based on stochastic processes. Annals of Mathematical Statistics 23, 193-212.

Balkema, A. A., and de Haan, L. (1974). Residual life time at great age. Annals of Probability 2, 792-804.

Basel Committee of Banking Supervision (2004). International convergence of capital measurement and capital standards; A revised framework. Technical Report, Bank for International Settlements, June.

Berg, D., and Aas, K. (2007). Models for construction of multivariate dependence. Technical Report, Norsk Regnesentral, Norwegian Computing Center, June.

Böcker, K., and Klüppelberg, C. (2006). Multivariate models for operational risk. Working Paper, TU Munich.

Chavez-Demoulin, V., and Embrechts, P. (2004). Advanced extremal models for operational risk. Technical Report, ETH Zurich.

Cherubini, U., Luciano, E., and Vecchiato, W. (2004). Copula Methods in Finance. Wiley, Chichester.

De Fontnouvelle, P., Rosengren, E. S., and Jordan, J. S. (2004). Implications of alternative operational risk modeling techniques. Working Paper, Federal Reserve Bank of Boston.

Degen, M., Embrechts, P., and Lambrigger, D. D. (2007). The quantitative modeling of operational risk: between g-and-h and EVT. ASTIN Bulletin 37(2), 265-291.

Delbaen, F. (2002). Coherent Risk Measures. Cattedra Galileiana. Scuola Normale Superiore di Pisa.

Di Clemente, A., and Romano, C. (2003). A copula-extreme value approach for modelling operational risk. Working Paper.

Dutta, K., and Perry, J. (2006). A tale of tails: An empirical analysis of loss distribution models for estimating operational risk capital. Working Paper, Federal Reserve Bank of Boston.

Eadie, W. T., Drijard, D., James, F. E., Roos, M., and Sadoulet, B. (1971). Statistical Methods in Experimental Physics. Elsevier Science Ltd, Amsterdam: North-Holland.

Embrechts, P., Furrer, H., and Kaufmann, R. (2003). Quantifying regulatory capital for operational risk. Derivative Use, Trading and Regulation 9(3), 217-233.

The Journal of Operational Risk

Volume 4/Number 3, Fall 2009 
Embrechts, P., Kaufmann, R., and Samorodnitsky, G. (2004). Ruin theory revisited: Stochastic models for operational risk. Risk Management for Central Bank Foreign Reserves, Bernadell, C., Cardon, P., Coche, J., Diebold, F. X., and Manganelli, S. (eds). European Central Bank, Frankfurt, pp. 243-261.

Embrechts, P., Klüppelberg, C., and Mikosch, T. (2002). Modelling Extremal Events for Insurance and Finance. Springer-Verlag, Berlin.

Embrechts, P., Lindskog, F., and McNeil, A. (2001a). Modelling dependence with copulas and applications to risk management. Handbook of Heavy Tailed Distributions in Finance, Rachev, S. (ed). Elsevier, Amsterdam, pp. 329-384.

Embrechts, P., McNeil, A., and Straumann, D. (2001b). Correlation and dependence in risk management: Properties and pitfalls. Risk Management: Value at Risk and Beyond, Dempster, M. A. H. (ed). Cambridge University Press, pp. 176-223.

Embrechts, P., and Puccetti, G. (2006). Aggregating risk capital, with an application to operational risk. The Geneva Risk and Insurance Review 31(2), 71-90.

Evans, J., Womersley, R., and Wong, D. (2007). Operational risk in banks: An analysis of empirical data from an australian bank. Working Paper, Institute of Actuaries of Australia.

Frachot, A., Moudoulaud, O., and Roncalli, T. (2004a). Loss distribution approach in practice. The Basel Handbook: A Guide for Financial Practitioners, Ong, M. (ed). Risk Books: London.

Frachot, A., Roncalli, T., and Salomon, E. (2004b). The correlation problem in operational risk. Operational Risk - Risk's Newsletter November.

Genest, C., and Favre, A.-C. (2007). Everything you always wanted to know about copula modeling but were afraid to ask. Journal of Hydrologic Engineering 12(4), 347-368.

Genest, C. and Rémillard, B. (2004). Tests of independence and randomness based on the empirical copula process. Test 13, 335-369.

Hill, B. M. (1975). A simple general approach to inference about the tail of a distribution. The Annals of Statistics 3, 1163-1174.

Huisman, R., Koedijk, K., Kool, C., and Palm, F. (2001). Tail-index estimates in small samples. Journal of Business and Economic Statistics 19(2), 208-216.

Joe, H. (1993). Parametric family of multivariate distributions with given margins. Journal of Multivariate Analysis 46, 262-282.

Koedijk, K. G., Schafgans, M., and de Vries, C. G. (1990). The tail index of exchange rate returns. Journal of International Economics 29, 93-108.

Mandelbrot, B. and Hudson, R. L. (2004). The Misbehavior of Markets: A Fractal View of Risk, Ruin and Reward. Basic Books, New York.

Mason, D. M. (1982). Laws of large numbers for sums of extreme values. The Annals of Probability 10(3), 754-764.

McNeil, A., Frey, R., and Embrechts, P. (2005). Quantitative Risk Management, Concepts, Techniques, Tools (Princeton Series in Finance). Princeton University Press, New Jersey.

Mikosch, T. (2006). Copulas: Tales and Facts. Extremes 9(1), 3-20. 
Moscadelli, M. (2004). The modelling of operational risk: experience with the analysis of the data collected by the Basel Committee. Temi di discussione (Economic Working Papers) 517, Bank of Italy, Economic Research Department, July.

Neslehova, J., Embrechts, P., and Chavez-Demoulin, V. (2006). Infinite mean models and the LDA for operational risk. The Journal of Operational Risk 1(1), 3-25.

Panjer, H. (2006). Operational Risk - Modeling Analytics. Wiley, Chichester.

Pickands, J. (1975). Statistical inference using extreme order statistics. Annals of Statistics 3, 119-131.

Savu, C., and Trede, M. (2008). Goodness-of-fit tests for parametric families of Archimedean copulas. Quantitative Finance 8(2), 109-116. 